\title{
Spectroscopy of T Tauri stars with UVES ${ }^{\star}$ Observations and analysis of RU Lup
}

\author{
H. C. Stempels and N. Piskunov
}

Department of Astronomy and Space Physics Box 515, 75120 Uppsala, Sweden

Received 25 February 2002 / Accepted 28 May 2002

\begin{abstract}
We present the first results of our observations of classical T Tauri Stars with UVES/VLT. The data consists of high signal-to-noise $(\geq 150)$ and high spectral resolution $(R \sim 60000)$ spectra. A large simultaneous wavelength coverage throughout most of the visible spectrum and comparatively short integration times allow us to study variability on short time-scales, using a number of diagnostics reflecting a wide range of physical processes. In particular we concentrate on the properties and geometry of the accretion process in the strongly accreting and highly variable CTTS RU Lup. We use the evolution of the level of veiling, the shapes of absorption and emission lines, and correlations between these diagnostics, to make new measurements of the fundamental stellar parameters as well as constraints on the accretion process and its geometry. We also derive the shortest time-scale of incoherent changes, which has implications for the nature of the accretion process in RU Lup.
\end{abstract}

Key words. accretion, accretion disks - stars: pre-main sequence - stars: individual: RU Lup

\section{Introduction}

Since their discovery one of the most intriguing properties of the classical T Tauri stars (CTTS) has been their apparently chaotic variability visible with nearly all observational techniques, from broad-band photometry to high-resolution spectroscopy. The spectra of these low-mass pre-main sequence objects are densely populated with emission lines including hydrogen, permitted and forbidden metallic lines and lines of helium (for a complete compilation of observational properties see e.g. Bertout 1989). The CTTS phase in the evolution of low-mass stars is rather short - a few million years. In the same age interval one finds, apart from CTTS, a group of weak line T Tauri stars (WTTS), with very weak or absent emission spectra. The characteristic properties of CTTS are attributed to the presence of an accretion disk while WTTS apparently lack such a disk. Whether there exists an evolutionary connection between the two groups is still unclear.

CTTS represent a real challenge for both modelling and observations. In this paper we will concentrate on the observational signatures of CTTS, on their variability and the plausible connection to the accretion processes.

The observational picture of CTTS is very complex. Photometry reveals a high level of variability on timescales from minutes to years with changes in brightness by a factor of two or more. CTTS show an excess of radiation in the IR

Send offprint requests to: $\mathrm{H}$. C. Stempels, e-mail: Eric.Stempels@astro.uu.se

* Based on observations collected at the European Southern Observatory, Chile (proposal 65.I-0404). (due the presence of the disk, e.g. Hartmann et al. 1994) and the UV (primarily contributed by the accretion shocks, e.g. Imhoff \& Giampapa 1980), which decreases the absorption line depth and makes the spectrum appear flatter than that of a main sequence star. This may in turn lead to erroneous spectral classification and fictitious reddening estimates. Several objects show a periodic component of the variability pattern which is associated with long-lived structures on the stellar surface, but there is no consensus about the nature of such structures.

The observed spectrum is dominated by hydrogen and iron emission lines. The strong resonance lines (e.g. Ca $\mathrm{H} \& \mathrm{~K}$ ) as well as lines of helium and forbidden lines of oxygen are seen in emission. Many of the stronger emission lines show absorption features in their blue wings, indicating a significant amount of material in stellar wind. Both the strength and the shape of most emission and absorption features are variable in time. In particular, the depth of the photospheric absorption lines appears to be smaller than for similar main sequence stars and it shows coherent changes in time. This was interpreted (e.g. Bertout et al. 1988) as excess continuum radiation which makes spectral lines appear shallower, the so-called veiling effect.

Circular polarization has recently been detected in the line profiles of He II lines by Johns-Krull \& Valenti (2000) and follow-up observations seem to point at a rather regular variability of the longitudinal field suggestive of rotational modulation. The polarization signal was also detected for some photospheric absorption lines (Valenti 2001).

The complex observational picture is in general agreement with the model of magnetospheric accretion introduced by 
Ghosh \& Lamb (1979) and later applied to T Tauri stars by Uchida \& Shibata (1985) and Königl (1991). In this model shearing within the disk transports angular momentum outward while the matter itself is accreted inward. In the inner regions, the magnetic field disrupts the accretion disk, which prevents matter from falling directly onto the equatorial regions of the stellar surface. Instead the material is channeled by the field lines away from the equatorial plane and falls in to high-latitude regions of the star forming hot shock regions. The strong UV and visible radiation from these regions produces the excess continuum observed as veiling, as well as Balmer jump emission, and possibly radiatively excites the emission lines formed in the infalling material. The several existing flavours of this model primarily differ in the way the field connects to the disk and how angular momentum is transported away from the star.

The most convincing evidence for the success of the magnetic accretion model is radiative transfer calculations for some of the emission lines (Muzerolle et al. 2001) which show qualitative and often quantitative agreement (despite multiple simplifications) with the observations.

Although the current model seems to be a good basis for understanding the physics of CTTS there are several important questions waiting to be answered. Most importantly:

- What exactly is the role of the magnetic field and what does the real magnetosphere look like?

- In what way do CTTS loose angular momentum while accreting disk material?

- What is the relation between CTTS and other groups of premain sequence objects of similar masses such as WTTS and FU Ori?

A single issue that may shed light on all these questions is the geometry of the accretion flow. Because realistic numerical simulations are beyond our current capabilities we are taking a semi-empirical approach; we plan to analyze the variability of the spectral features formed in the circumstellar medium. The approach is not fundamentally new; e.g. Doppler Imaging has successfully been applied to reconstruct 2D maps of the surface distribution of active regions on late-type stars (see Strassmeier et al. 1991) and of the chemical composition and magnetic fields on CP stars (see Kuschnig et al. 1999). This well-established technique recovers the spatial information from rotational modulation of spectral line profiles. A similar approach is Doppler tomography, which is extensively used for studying the geometry of the accretion disks in cataclysmic binary systems (Marsh \& Horne 1988).

Our intention to reconstruct the accretion geometry in CTTS is a much bigger challenge because we have to deal with three-dimensional structures, unknown sources of variability and uncertain information about the physical conditions in the circumstellar medium. Therefore, we plan to achieve this goal in three steps: (1) obtaining a dense time series of highresolution and high $S / N$ observations of CTTS, (2) developing a reconstruction technique and testing it using numerical experiments and, finally, (3) applying it to the real data.

In this paper we describe the results of the first step. In the following two sections we describe our data set obtained at the VLT and the data reduction. Next we take a closer look at the different accretion and wind diagnostics present in the spectra and compare them with the general model of CTTS outlined above. This information will serve as a starting point for the next step: reconstruction of the accretion geometry, which will be the subject of subsequent papers.

\section{Observations of CTTS}

\subsection{Previous observational studies of TTS}

The characteristics of CTTS have been studied with many different observational techniques. Below we list some of the recent analyses of CTTS.

The general model of a CTTS in terms of a chromospherically active star with an accretion disk was put forward by Bertout et al. (1988). They modeled CTTS with narrow band photometry throughout the entire accessible spectral range, from UV to IR, by separating the energy source in three components; a star, disk, and a boundary layer. It is clearly shown that the disk models of Lynden-Bell \& Pringle (1974) can be successfully used to explain the main observational properties of CTTS and to determine the accretion rates. They also noticed that observations of Balmer line profiles are not in agreement with the expected profiles of their model of equatorial accretion.

The shapes of emission lines of hydrogen, but also of helium, oxygen, sodium and calcium, were studied at high spectral resolution (resolving power $R \sim 35000$ ) by Muzerolle et al. 1998b). They used these lines as diagnostics of the accretion shock region, which is assumed to be the formation region of these lines. In order to explain the observed spectral line shapes, they used the model of infall along a dipole-shaped magnetic field (Muzerolle et al. 1998a), with an (implicitly assumed) isotropic and time-independent accretion flow.

Gullbring (1994) compared the polar accretion model versus the equatorial accretion model using high-speed photometric observations through the IR, optical and UV. His observations favoured the polar accretion model. In a later paper, Gullbring et al. (1996) obtained 51 high-resolution spectra $(R \sim 26000)$ of the CTTS BP Tau in two observing periods. They analyzed the time evolution of the shapes of the strongest emission lines $(\mathrm{H}, \mathrm{He}, \mathrm{Na}$ and $\mathrm{Ca}$ ) with respect to velocity offset. The spectral asymmetry in these variations was interpreted as an indication that the accretion flow is variable and controlled by a magnetosphere that is inclined relative to the rotational axis.

The time evolution of the strongest emission lines was investigated in detail by Smith et al. (1999) with long-slit spectroscopy $(R \sim 750)$ of 6 CTTS in the optical wavelength range over a period of 5 days. They obtained spectra with relatively short integration times of 5-15 min. This allowed the correlation between the dominating emission features to be closely followed, and, knowing the time scales, some sources of variability to be identified. The shortest time scale of variability found was of the order of hours, which they relate to magnetic flaring. They also relate a sequence of changes in the shapes of 
different spectral lines to the transition of an active accretion region over the stellar limb (rotational modulation).

Calvet \& Gullbring (1998) investigated in detail the physical conditions in the accretion shock and its consequences on the veiling effect, putting emphasis on determining its wavelength dependence and the corresponding filling factors of the accretion regions on the visible stellar disk. They compared their shock models with observations of CTTS from Gullbring et al. (1998) and found that the filling factor of typical CTTS is of the order of a few percent (see Sect. 4.2). However, no attempt was made to study the time evolution of the veiling like Johns-Krull \& Basri (1997), who derived the veiling changes using 117 spectra with $R=48000$.

Hartigan et al. (1995) calculated accretion rates by equating the excess bolometric luminosity to the amount of matter in the accretion flow, as well as outflow rates by studying the line luminosities of forbidden spectral lines formed in the wind. They point out that forbidden emission lines in the spectra of CTTS seem to consist of two components: a low- and a highvelocity component. The high-velocity component is identified with a stellar jet, while the low-velocity component, originating from a much more extended region, corresponds to a disk wind. Kwan \& Tademaru (1995) investigated these forbidden lines to constrain the physical location and mechanism of wind generation. Both papers find the ratio of in- to outflow rates to be at least a factor of 10. Lamzin et al. (2001) derived accretion rates (or the upper limits) by comparing the computed strength of a number of coronal lines that should be produced in the post accretion shock zones on T Tauri stars with observations.

Doppler Imaging has been used extensively to determine the location and evolution of active regions on the surfaces of low accretion TTS (see Johns-Krull \& Hatzes 1997; Rice \& Strassmeier 1996). These studies revealed a series of dark and bright spots located at high latitudes consistent with the magnetospheric accretion model. They also find that the active regions do not cover the stellar surface in distinct circular regions as would be expected in the case of continuous axisymmetric accretion controlled by a dipolar field. On the other hand, because of the low accretion rate, chromospheric activity may be dominating the surface structures.

Direct detection of the magnetic field has been attempted with polarimetry of spectral lines that are sensitive to the Zeeman effect. Basri et al. (1992) estimated the strength of the magnetic field of WTTS of the order of $1 \mathrm{kG}$. However, the uncertainties were large. New measurements of circular polarization show several kGauss fields on a number of CTTS derived from the line profiles of high-excitation helium lines and some photospheric absorption lines (Johns-Krull \& Valenti 2000). A realistic attempt to reconstruct the magnetic field vector distribution over the surface of a TTS is still waiting for high-precision 4-Stokes parameter observations.

\subsection{Our observations}

During the nights of 16th and 17th of April 2000 we obtained spectra of a number of $\mathrm{T}$ Tauri stars. The observations have been performed with the Ultraviolet Visual Echelle
Spectrograph (UVES, D'Odorico et al. 2000) at the VLT Kueyen telescope in Chile. The selection of the instrument and observing mode was determined by a number of goals. In particular, we required:

- High signal-to-noise ratio to be able to analyze the shape of emission and absorption lines even superimposed a strong emission background. With typical veiling values of the order of 10 , even the detection of a line which is $10 \%$ deep relative to the stellar continuum requires a signal-to-noise ratio of 100 .

- High spectral resolution in order to study different parts of the accretion flow corresponding to different radial velocities and to be able to detect discontinuities if the accretion consists of large individual parcels of material. Such parcels, if they exist, would produce individual line emission features in the spectrum with a typical $F W H M$ of a few $\mathrm{km} \mathrm{s}^{-1}$ which would be possible to detect with UVES resolution of $R \approx 60000$. A high resolution is also required to study fine structure of emission lines such as broad and narrow components and the velocity structure of the stellar wind.

- Large simultaneous wavelength coverage in order to probe a wide range of spectral lines, reliably derive fundamental stellar parameters, properties of veiling and general characteristics of the circumstellar medium. Simultaneous measurements throughout the visible spectrum are critical for a robust estimate of veiling and for finding correlations between different features.

- Short integration times and dense time series in order to measure spectral variability and establish the time scale of coherent changes.

- Sufficiently long observing run in order to distinguish between rotational modulation and variability of the mass accretion rate. Unfortunately this condition was not fulfilled due to the high pressure on UVES time.

The UVES/VLT is a perfect combination for this task. An amazingly high throughput allows a high SNR $(\geq 150)$ for a $V=12^{\mathrm{m}}$ star to be obtained in an exposure as short as $10 \mathrm{~min}$ utes with $R \approx 60000$. A very important property of UVES is the ability to observe simultaneously with the red and the blue arm, though with different integration times, in order to obtain the right compromise between the integration time and the SNR. Also, the instrument gives nearly continuous coverage from $3500 \AA$ to $6700 \AA$.

The weather was good, except for one hour of strong winds during the first night, followed by a few hours of bad seeing. This was compensated for by using an image slicer and opening up the slit from 0.6 to 0.7 arcsec, degrading the spectral resolution from 60000 to 50000 in favour of obtaining a better SNR. In general, the acquired SNR was better than expected, mostly due to the fact that the mirrors were aluminized just two weeks before our observations took place. We used a so-called UVES special setting, which allowed us to cover the spectra continuously from $3500 \AA$ to $6700 \AA$. Even with a high spectral resolution, we could for the brightest stars obtain a time sampling of $600 \mathrm{~s}$ through the red arm. 


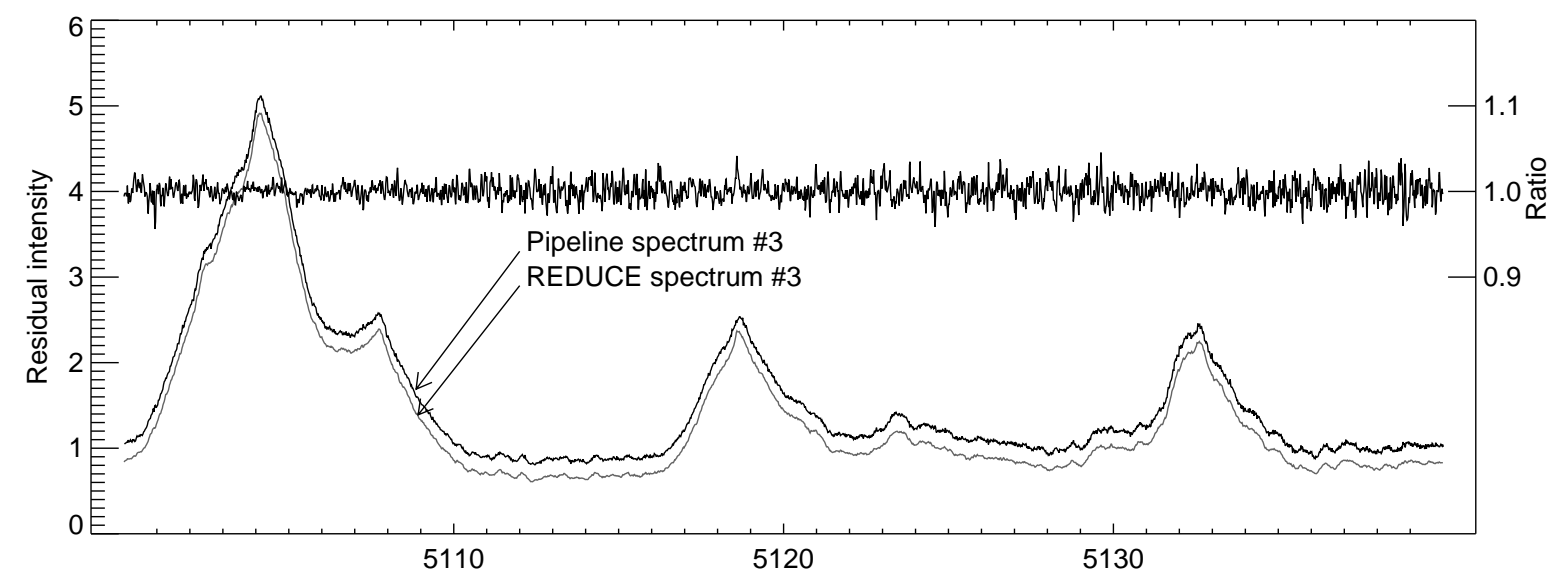

Fig. 1. Comparison between a spectrum of RU Lup reduced with the UVES pipeline (Ballester et al. 2000, upper darker line) and with the REDUCE package (Piskunov \& Valenti 2002, lower lighter line). For clarity, the pipeline spectrum is shifted upward by 0.2. Overplotted is the ratio of the two reduced spectra (Scale on the right vertical axis). We see no correlation between the ratio pattern and the spectral features.

\section{Data reduction}

The UVES data was processed using the spectroscopic data reduction package REDUCE (Piskunov \& Valenti 2002) which combines a very robust order location procedure with $2 \mathrm{D}$ modelling of individual orders to perform optimal extraction, correct for cosmic rays and remove scattered light. The package is written in IDL and has been used for processing a variety of CCD spectroscopic data. Figure 1 shows the comparison of a spectrum fragment extracted with REDUCE and the UVES pipeline demonstrating the excellent quality and consistency of both packages.

The reduction procedure starts with the construction of master bias and master flat field frames for each night. For the master bias and master flat we typically used 12 and 10 frames respectively by developing statistical models for each pixel and rejecting the outliers. After the echelle orders had been located a novel spatial profile decomposition algorithm was used to construct a $2 \mathrm{D}$ model of the master flat. The master flat was normalized with the model in order to keep pixel weights consistent with the accumulated counts. Next, science frames were flat-fielded and optimally extracted. The optimal extraction procedure is also based on slit function decomposition, allowing us to correct bad pixels, including CCD defects and cosmic ray spikes, in the science frames. The extracted $1 \mathrm{D}$ spectra were normalized by their corresponding blaze functions, wavelength calibrated and reduced to heliocentric velocity. Finally we produced complete spectra by means of order splicing, only individual orders were used in the analysis in this paper.

\section{RU Lup}

The acquired dataset is very large and consists of many different types of targets. It is beyond the scope of this paper to present a detailed analysis of all the data or to develop a model explaining the observations. In order to assess the quality of the data and to identify critical diagnostics of the geometry of the accretion process we mainly concentrated on RU Lup, the star for which we obtained the most extensive dataset.

\subsection{Properties of RU Lup}

RU Lup is one of the most active CTTS with very strong emission and variability in Balmer lines and other diagnostics. Strong veiling makes it difficult to determine stellar parameters, but our best estimates for the fundamental parameters (see also Sect. 4.3) are $T_{\text {eff }}=3950 \mathrm{~K}, \log g=3.9$, $L \approx 0.49 L_{\odot}, R=1.7 R_{\odot}$ and $M \approx 0.8 M_{\odot}$. From our data we measured the $v \sin i$ of RU Lup to be $9 \pm 0.9 \mathrm{~km} \mathrm{~s}^{-1}$ and $v_{\text {rad }}=-1.9 \pm 0.02 \mathrm{~km} \mathrm{~s}^{-1}$. Under the assumption of corotation of the star and the inner boundary of the accretion disk, Lamzin et al. (1996) estimated the rotational period to be 0.8 days. Attempts to use circular polarimetry to measure the global magnetic field resulted in an upper limit of $500 \mathrm{G}$ (Johnstone \& Penston 1987). The estimated accretion rate is $\dot{M} \approx 3 \times 10^{-7} M_{\odot} \mathrm{yr}^{-1}$, assuming $A_{V}=0.3^{\mathrm{m}}$ and $d=200 \mathrm{pc}$ (Lamzin et al. 1996, but see also comments by Bertout et al. 1999).

\subsection{Veiling}

The presence of a veiling pseudo-continuum in CTTS was discovered as early as T Tauri stars themselves (Joy 1945). According to current models, the veiling continuum originates in the accretion shock region on the stellar surface and decreases the depth of stellar absorption lines.

Because the veiling continuum is closely related to the accretion process and particularly the instantaneous accretion rate, veiling is an important diagnostic of the accretion activity of CTTS. The relation between veiling and accretion in CTTS was discussed in detail by Calvet \& Gullbring (1998). Changes in the level of veiling are a diagnostic of the size of the accretion region, which in turn allows the nature of variability in the accretion flow to be constrained.

The veiling factor $V$, by which the pseudo-continuum from the hot accretion shock decreases the line depth of photospheric lines, is defined as:

$V=I_{\mathrm{pc}} / I_{\mathrm{sc}}$ 

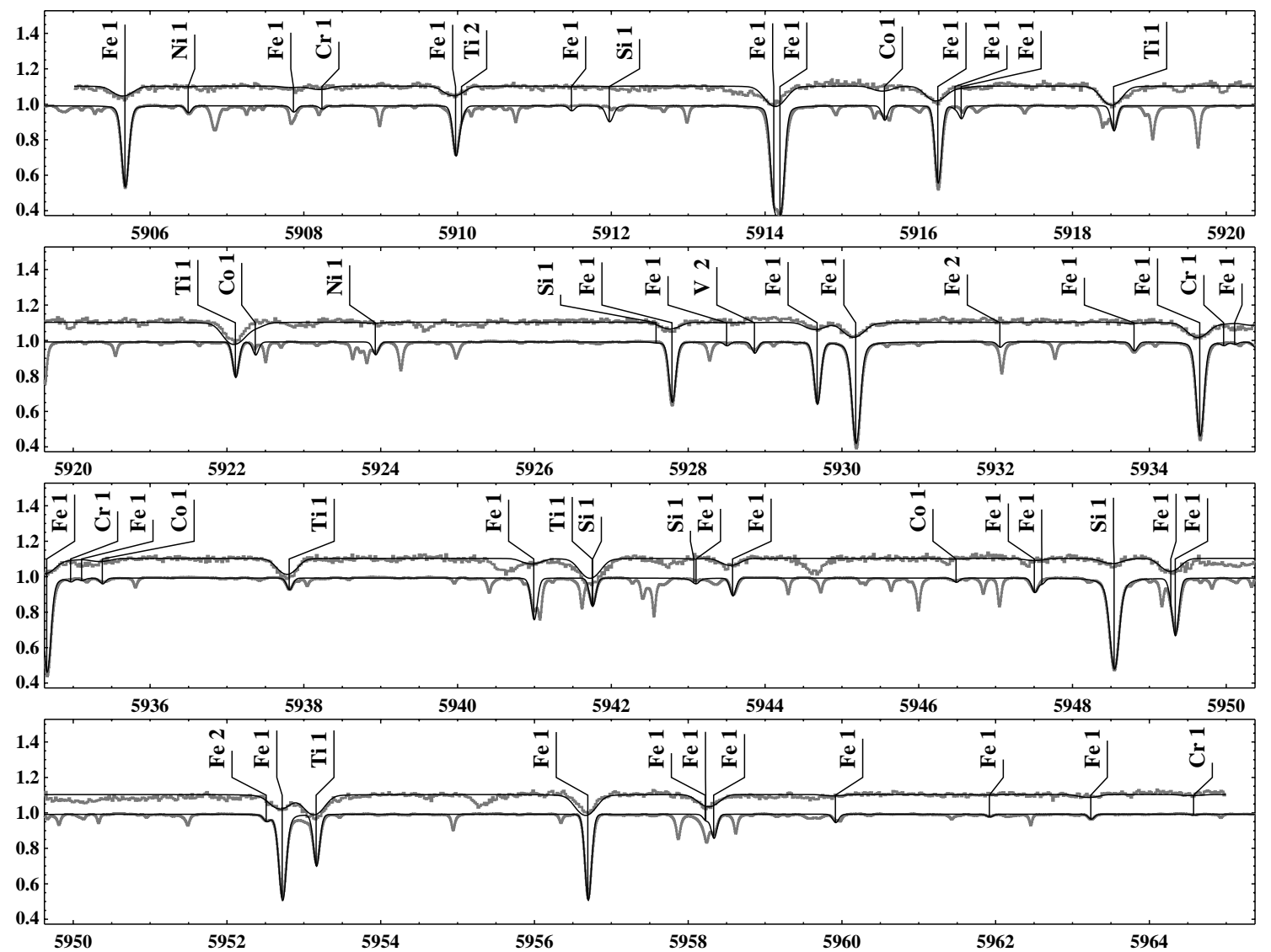

Fig. 2. SME fit to the NSO Solar spectrum (lower two lines) and to the RU Lup spectra (upper two lines) in the 5905-5965 A region. For fitting the solar spectrum we used the MARCS solar model (see Asplund et al. 1997 for more details) and a VALD line list. No parameter adjustment was performed for the Sun. The same line list was then used to fit the RU Lup spectra (all at once) searching for the optimal combination of $T_{\text {eff }}, \log g, v \sin i, v_{\text {rad }}$ and veiling. Veiling was adjusted separately for each observation.

where $I_{\mathrm{sc}}$ is the stellar continuum intensity, and $I_{\mathrm{pc}}$ the intensity of the pseudo-continuum. Then the observed relative line depth $d^{*}$ can be expressed in terms of the unveiled relative line depth $d$ as:

$d^{*}=\frac{d}{1+V} \rightarrow V=\frac{d-d^{*}}{d^{*}}$.

It is important to realize that in CTTS the veiling effect in essence is the result of the sum of two spectra, where the excess continuum, in the visual range, can be described (at least as a first approximation) by a black body spectrum scaled with the surface filling factor (Gullbring et al. 1998). Assuming that the shape of the intrinsic stellar spectrum is known (see below), the shape of the veiling continuum will be a measure of the temperature of the accretion shock. Such a model also provides a diagnostic of the surface filling factor of the hot accretion shock, because the veiling factor is proportional to the visible area of the shock emission. As the filling factor affects all the wavelengths in the same way, it can be determined simultaneously with the temperature of the shock.

\subsubsection{Measuring the veiling}

We present two methods we used to determine the veiling in our set of observations of RU Lup. Because veiling is caused by a hot pseudo-continuum superimposed on a relatively cool stellar spectrum, it is not unreasonable to assume that the veiling factor does not change over a short spectral interval. Both methods use this assumption and are based on least-squares fitting; that means we search for the minimum of:

$\chi^{2}=\sum_{\lambda, \phi}\left\{d^{*}-\frac{d}{1+V}\right\}^{2}$.

The first method assumes that the intrinsic line depth $d$ is known, for example from a synthetic spectrum or a template star. Then, one can determine the most likely $V$ for a region of the spectrum by minimizing Eq. (3) with respect to $V$. This is done individually for each observation $\phi$.

A second method is based on the assumption that the intrinsic unveiled spectrum does not change from observation to observation. Then it is possible to treat the unveiled line depth $d$ as a free parameter instead of assuming an artificial or template spectrum. The introduction of another free parameter can be compensated for by summing over all observations $\phi$, and minimizing Eq. (3) with respect to both $V$ and $d$. The minimum is found by iteratively solving the system of equations for $d_{\lambda}$ and $V_{\phi}$ arising from the usual condition that the partial derivatives in the minimum be equal to zero. It is important to realize that for this method, because we did not make any assumption about the unveiled line depth $d$, there is a degeneracy 


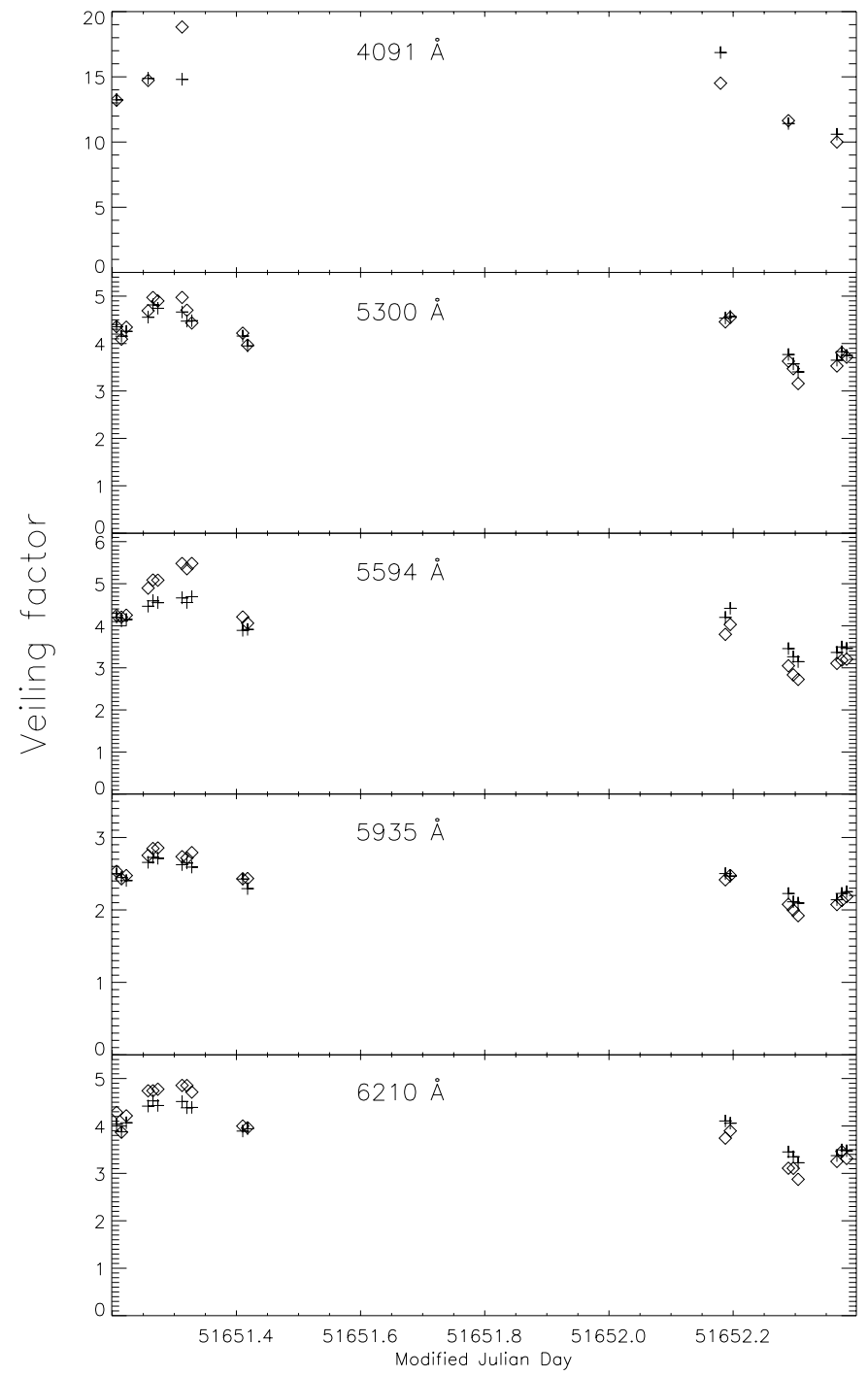

Fig. 3. Evolution of the veiling factor of RU Lup during the observing run. Diamonds refer to the first method, while crosses refer to the second method. The results of the second method only reflect relative changes in the veiling factor (see text) and are therefore scaled to have the same average veiling value. This has no impact on the evolution of the veiling and the agreement between the two methods is good.

between $d$ and $1+V$. Any increase of $d$ can be compensated by a corresponding increase of $1+V$. In other words, we cannot determine the zero point for the veiling.

Both the first and the second method have their advantages. The first method offers absolute veiling measurements, but requires knowledge of the intrinsic spectrum. Such a spectrum may not always be available. The second method can be applied without a priori knowledge of the spectral region, but offers only relative veiling measurements. The combination of the two methods provides us with the best veiling measurements and allows to derive the shock temperature and the filling factors. We determined the veiling at five selected wavelengths with both methods. The results presented in Fig. 3 show rather smooth variations during a single night of observations.

Also Hartigan et al. (1989) and Calvet \& Gullbring (1998) calculated the veiling factor in a number of T Tauri stars. Both

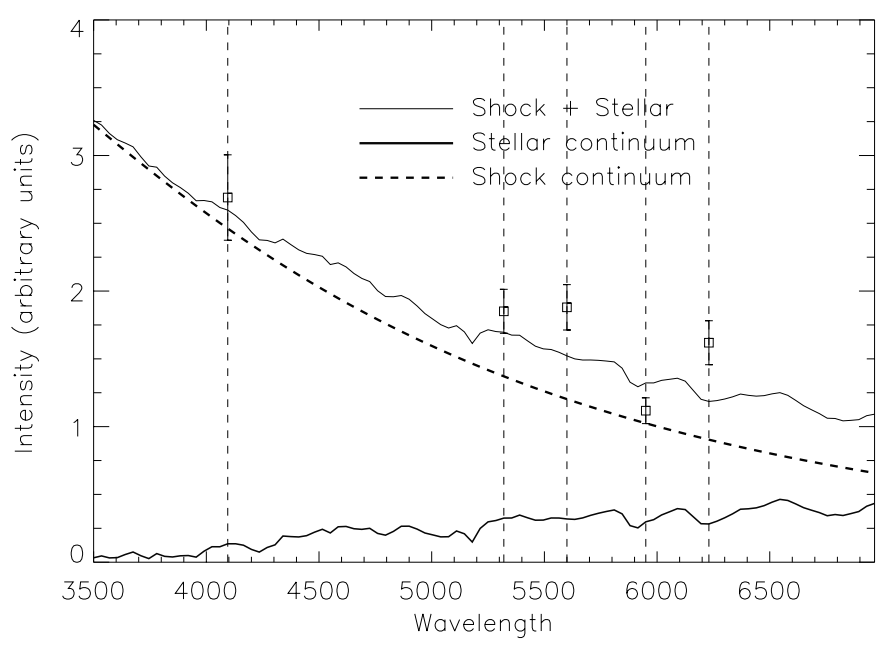

Fig. 4. Example of a least-squares fit according to Eq. (4) of the filling factor $f f$ and the pseudo-continuum temperature $T_{\mathrm{pc}}$ on top of a stellar continuum corresponding to $T_{\mathrm{sc}}=4000 \mathrm{~K}$.

methods are in essence similar to our first method, because they use template spectra for the underlying photosphere when fitting.

\subsubsection{Two-component model}

As was mentioned above, the wavelength dependence of the veiling can provide estimates of the temperature and filling factor of the accretion regions. In order to determine these parameters, we developed a simple two-component model. In this model, we approximate the veiling continuum contribution with a black-body function, and the stellar contribution with a synthetic flux spectrum based on the fundamental stellar parameters we derived for RU Lup. This can be expressed as follows:

$F_{\text {tot }}=\left(1-S_{\mathrm{ff}}\right) \cdot F_{\mathrm{star}}+S_{\mathrm{ff}} \cdot \pi B_{\lambda}\left(T_{\mathrm{pc}}\right)$

where $F_{\text {star }}$ is the astrophysical flux of the stellar component, $T_{\mathrm{pc}}$ the temperature of the pseudo continuum, and $S_{\mathrm{ff}}$ the filling factor.

With this model in mind, the evolution of the temperature and filling factor of the accretion regions was determined by a least-squares fit of the veiling measurements we obtained at five different wavelengths to a scaled black-body function superimposed on a stellar flux spectrum. For each observation we determined the most likely $T$ and $S_{\mathrm{ff}}$ by minimizing

$\chi^{2}=\sum_{i}\left\{w\left(\left(\frac{F_{\mathrm{tot}}\left(T_{\mathrm{pc}}, S_{\mathrm{ff}}\right)}{F_{\mathrm{star}}}-1\right)-V_{i}\right)\right\}^{2}$

where the summation is taken over the five wavelengths where the veiling $V_{i}$ was determined with Eq. (3). The weight $w$ is defined as the inverse of the error of Eq. (3), which is $1 /\left(2 \cdot \nabla \chi^{2}\right)$. An example of such a fit is shown in the upper panel of Fig. 4. However, because of how $T_{\mathrm{pc}}$ and $S_{\mathrm{ff}}$ enter Eq. (5), the values of the parameters $T_{\mathrm{pc}}$ and $S_{\mathrm{ff}}$ are strongly correlated; an increase in the veiling temperature can be compensated by a decrease in the filling factor. To illustrate this effect, we performed full error propagation and calculated $\chi^{2}$ for a range of 


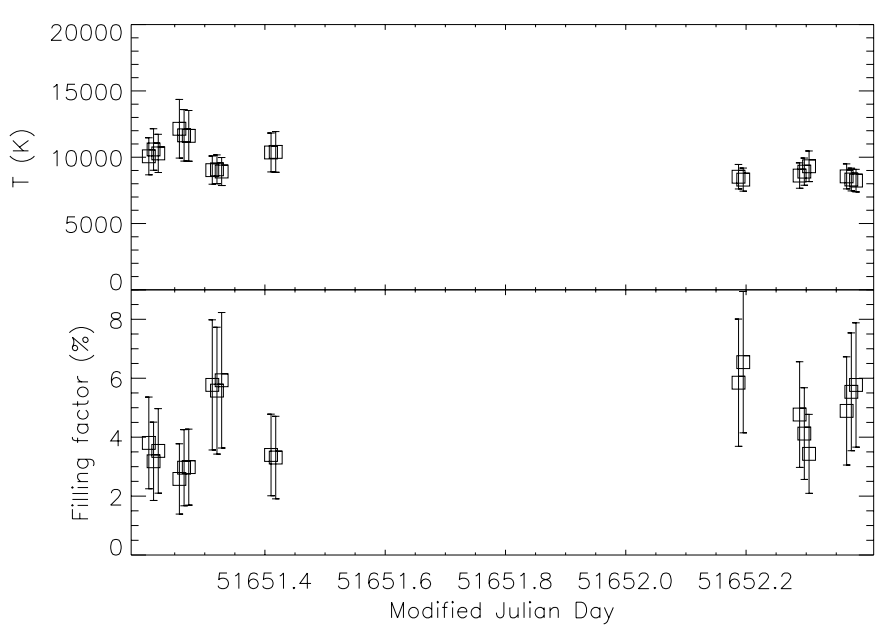

Fig. 5. Evolution of temperature and filling factor during the observation run. The errorbars assume that the temperature and filling factor are independent variables (not true, see text and Fig. 6).

$T_{\mathrm{pc}}$ and $S_{\mathrm{ff}}$ to obtain confidence regions of the filling factor and temperature; we show two such confidence regions in Fig. 6. Because the confidence regions of the individual observations do not overlap, it is clear that the changes in the evolution of the temperature and filling factor of the accretion region are significant.

\subsubsection{Interpretation of veiling measurements}

In Fig. 5 we show a time-series of the derived temperatures and filling factors during our observation run. The quoted errorbars may be slightly misleading, because they must be interpreted with help of Fig. 6. Although the error bars show the $1 \sigma$ confidence limit for each parameter, as if the parameters were independent, detectable variations, though correlated, occur on a much smaller scale. The veiling measurements we obtained serve as a good starting point for describing the accretion activity of RU Lup throughout our observing run. From Fig. 5 one can see that an increase in the overall veiling continuum occurred in the middle of the first night. From here on, we shall refer to such an increase as an accretion event, even if we cannot determine that the cause of such an event is an increase of the accretion area or an enhancement of the accretion flow to the star. The second night starts with a small (declining) accretion event followed by the onset of a new accretion event.

Calvet \& Gullbring (1998) calculated models of CTTS accretion shock regions and the corresponding emerging energy flux, and compared these with observed accretion rates. They found that the temperature of the shock region of CTTS is approximately $10000 \mathrm{~K}$. They also report typical values for the filling factor of CTTS to be between $0.1 \%$ and $1 \%$. This they interprete as evidence that the accretion flow of CTTS is not continuous and axisymmetric, because, if the accretion flow were continuous and axisymmetric and would follow the field lines of a (possibly inclined) dipole, the projected surface area that should be covered is of the order of $10 \%$.

As can be seen from Fig. 5, we find the temperature in the shock region and the filling factor of the order of $10000 \mathrm{~K}$ and

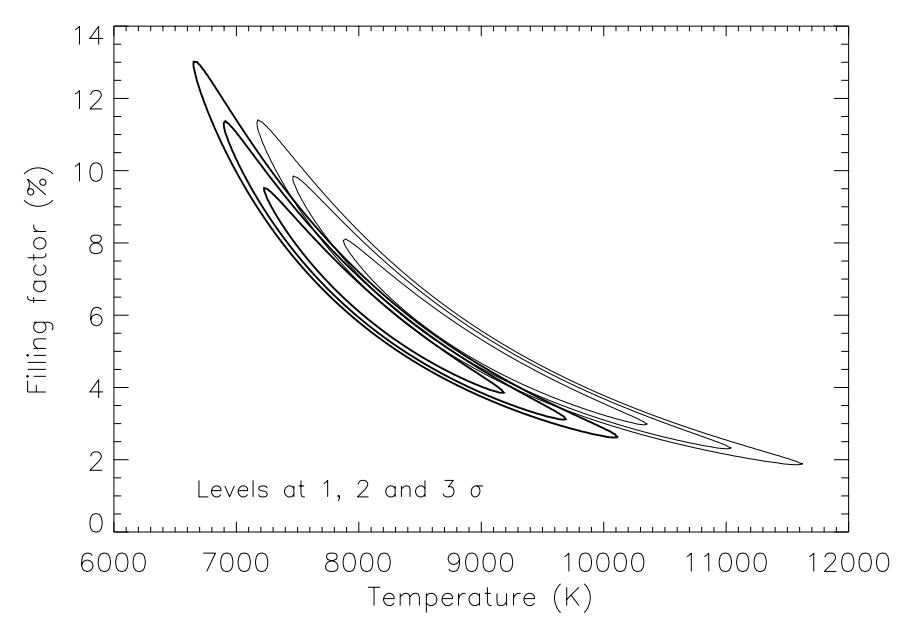

Fig. 6. Examples of confidence regions in parameter space of the temperature and filling factor for two observations of the second night. contours are denote the 1, 2 and $3 \sigma$ confidence intervals. The absence of overlap indicates significant changes in the temperature and filling factor.

$4 \%$ respectively. As RU Lup is known to have a high accretion rate (up to $10^{-6} M_{\odot} \mathrm{yr}^{-1}$, see Gullbring et al. 1998 and Lamzin 2001), it is not surprising that RU Lup seems to show a filling factor that is higher than the average. The evolution of the filling factor correlates with the accretion activity, indicating that either the visible area of the accretion region changes due to projection effects, or the total area of the regions changes due to a time-dependent flow.

Changes in the filling factor may be related to the changes in the accretion rate. Ardila \& Basri (2000) found that the accretion rate correlates with the filling factor, and that this relation is not due to geometrical effects. This implies that the observed variability in RU Lup is caused by inhomogeneous accretion.

\subsection{Typical metallic lines}

Metallic absorption lines allow the effective temperature, surface gravity, metallicity and rotational velocity of the central star to be determined. Special care must be taken to "unveil" the spectra and to avoid regions with a significant contribution of emission components. In order to establish the presence of line emission we used spectral synthesis. In the first step we verified the line lists extracted from the Vienna Atomic Line Database (VALD, see Piskunov et al. 1995) against the NSO solar atlas (Kurucz et al. 1984). One of the best regions for determining fundamental stellar parameters was found between 5905 and $5965 \AA$ where the atomic data corresponds rather well to the solar spectrum and the spectra of RU Lup have no obvious emission components associated with metallic lines. We still see some contribution from emission by $\mathrm{H} \alpha$, but this is small and featureless, time-independent, and does not have any impact on the line shapes. In the last step we fitted the observations with the synthetic spectrum by adjusting the veiling, the effective temperature, rotational and radial velocity. The veiling was adjusted for each observation while other parameters were assumed to be constant. We used a modification of the SME code 
(Valenti \& Piskunov 1996) to achieve the best fit. The modification consists of replacing the fit of the continuum level with a two-parameter fit of the veiling. We also looked at the possibility to significantly improve the values of the surface gravity and turbulent velocities but the quality of our data does not allow very accurate values to be obtained. No deviations from mean solar abundance were required. The resulting parameters for this region of the spectrum are $T_{\mathrm{eff}}=3950 \mathrm{~K}, \log g=3.9$, $v \sin i=9.1 \pm 0.9 \mathrm{~km} \mathrm{~s}^{-1}$ and $v_{\text {rad }}=-1.9 \pm 0.02 \mathrm{~km} \mathrm{~s}^{-1}$. The final fits to the 5905-5965 $\AA$ spectra of the Sun and RU Lup are shown in Fig. 2.

Metallic emission lines are good tracers of the structure of the accretion flow, because these lines form under conditions where the temperature and pressure are relatively low. A curveof-growth analysis (Ti II, Ca II, Fe I \& II) and more detailed escape probability calculations (Gahm et al. 1974) show that the emission component of the metallic lines are mostly optically thin. This is confirmed by the fact that photospheric absorption lines are often seen superimposed on emission components. Although the detailed level population calculations are very difficult due to the unknown geometry it is still possible to derive some properties of the line forming regions by comparing emission lines with common levels.

Figure 7 shows the observed line profiles for a number of Fe I and II lines. The left panels compare relatively unblended lines sharing the same upper level. For both ions we included the strongest transitions that fall in our spectral region. We can see that in general the line shapes of the dominating transitions are very similar. When comparing line shapes of transitions from a common upper level, one finds, after rescaling by the corresponding Einstein $A_{\mathrm{ul}}$ transition probabilities, that the weakest lines are indeed formed under optically thin conditions. The strongest lines show some signs of saturation, but are still formed in a close to optically thin environment. An example of the latter case is shown in the lower left panel of Fig. 7. The middle panel compares lines with the same lower level. Again we note the amazing resemblance of the shape and width of the profiles. Using Gauss decomposition of these profiles, we find that the majority of the profiles can be reasonably approximated by three emission components. We find a narrow $\left(F W H M \sim 20 \mathrm{~km} \mathrm{~s}^{-1}\right)$ central component superimposed on two broader $\left(F W H M \sim 100 \mathrm{~km} \mathrm{~s}^{-1}\right)$ components offset by approximately +50 and $-50 \mathrm{~km} \mathrm{~s}^{-1}$. Although the relative strength of the latter two components varies from line to line, the similarity in the decomposition is striking.

An alternative explanation for the narrow core is the presence of absorption components. For example, the profile of Fe II could be reproduced by one photospheric component centered on the stellar radial velocity (as clearly shown by weaker lines in Fig. 7), and one blueshifted component at $-20 \mathrm{~km} \mathrm{~s}^{-1}$. However, this would mean that absorption lines of Fe II should also be seen in the photospheric spectrum of RU Lup. Because this is not the case, we reject this alternative explanation.

The right panels of Fig. 7 compare line profiles throughout the whole spectral range. We note the gradual change from blueshifted to redshifted excess emission as we move to longer wavelengths. Although the exact shape is different for different lines, the resemblance between lines throughout the observed spectral range is remarkable both for neutral and ionized spectra. Even more amazing is the resemblance between the shape of the neutral and ionized lines. We conclude that the Fe emission lines are optically thin that and the exact shape is defined by the velocity field.

\subsection{Forbidden lines}

In regions with low temperatures, located relatively far away from the central star, line excitation occurs mostly though radiation from the star and the accretion shock region rather than through collisions, causing an overpopulation of excited states. Such an environment occurs in the accretion flow and in the wind of CTTS. By studying forbidden lines originating in these regions one can probe their geometrical structure and physical conditions. The strongest forbidden emission lines in CTTS are [O I] at 5577, 6300, and $6363 \AA$, [S II] at 6731 and $6716 \AA$, and [N II] at $6583 \AA$. All these lines can be found within the covered wavelength range of our sample. However, we do not see any emission or absorption at the position of [N II] at $6583 \AA$. No variation in the line emission of [S II] at $6731 \AA$ was detected.

In Fig. 8, we present a time-series of the [O I] 6300 and $6363 \AA$ lines. Emission of [O I] is well visible, and due to the apparent radial velocity the telluric emission components do not overlap with the stellar profile. We do see a number of stellar absorption lines superimposed on the profile, confirming that this line is formed on optically thin conditions.

As can be seen from Fig. 8 the shape of the [O $\mathrm{I}$ ] lines is asymmetric. Redshifted emission is absent, while the blue wing consists of a strong component at low velocity and a broad shoulder towards higher velocities $\left(200 \mathrm{~km} \mathrm{~s}^{-1}\right)$. This may be interpreted as a combination of a steady and wide outflow, combined with an accelerated and collimated polar jet. The former component is either a disk wind or hot gas located above the polar accretion areas.

Variability of these profiles is small, as should be the case for time-integrated signatures of outflow. On the other hand, there is some correlation with accretion events. The $6300 \AA$ line, and in a less strong fashion also the $6363 \AA$ line, shows an increase of emission in the low-velocity component a few hours after the accretion events. The low-velocity component is tentatively identified as the base of a collimated jet; the high-velocity component of the jet does not show variation because the time-integrated nature of the optically thin line destroys signatures of individual events.

\subsection{The strongest emission lines}

Within our wavelength coverage we have a complete set of observations of Balmer lines. Other strong optical emission lines present in our sample are $\mathrm{He}_{\mathrm{I}}$ at $5876 \AA$, the $\mathrm{Na}$ I $\mathrm{D}$ doublet at 5890 and $5896 \AA$ and the $\mathrm{Ca}$ II H \& K lines at 3968 and $3934 \AA$. The presence of strong emission from lines with high excitation potentials (such as He I $5876 \AA$ ) suggest high temperatures in or near the accretion shock. Though strong, the large intrinsic width of these lines prevents investigating the 

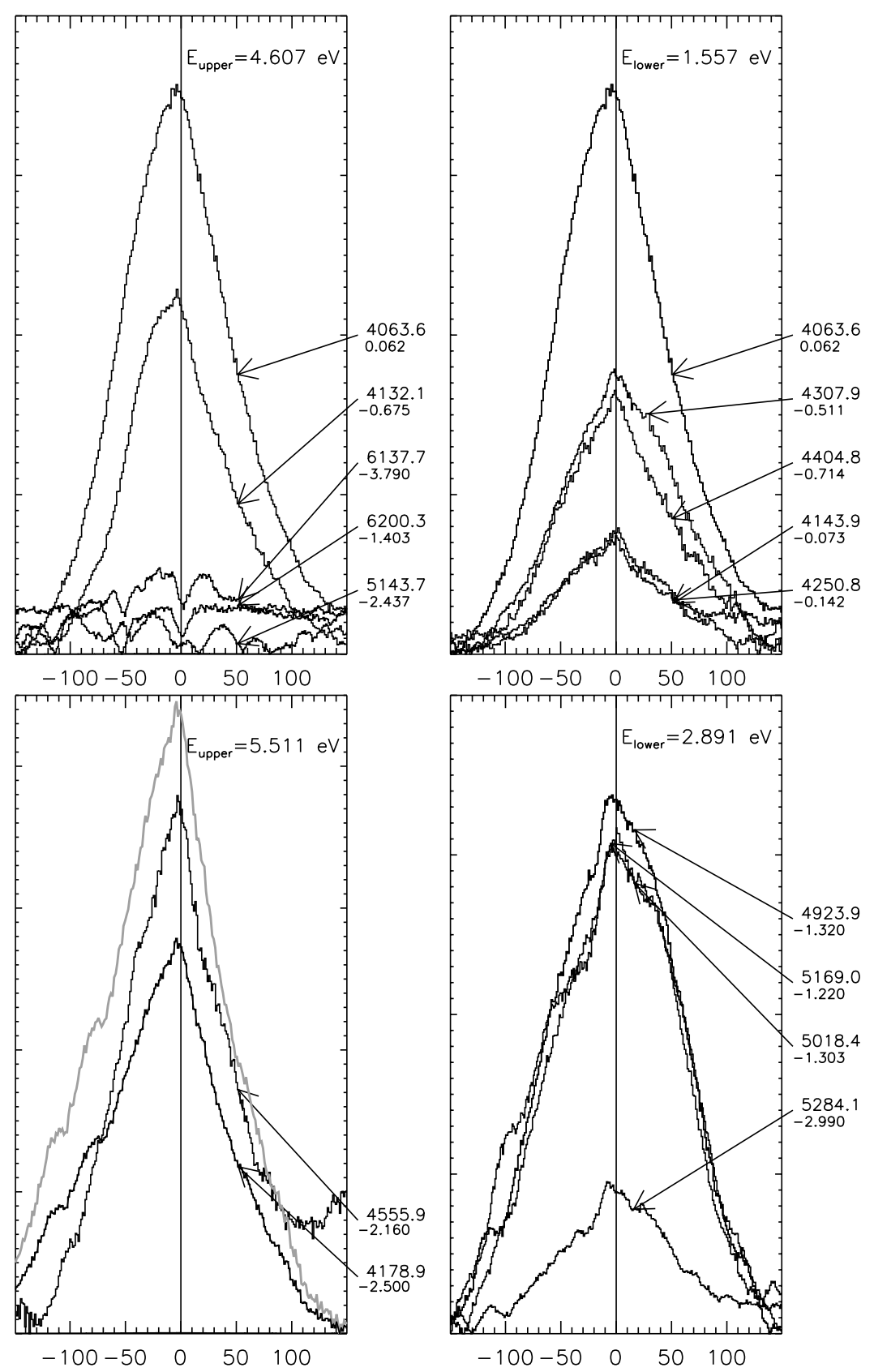

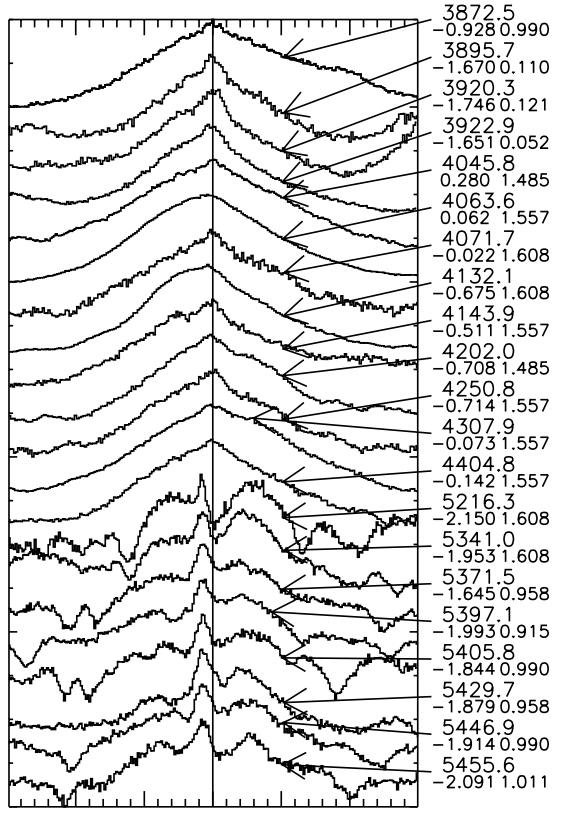

$-100-50 \quad 0 \quad 50 \quad 100$

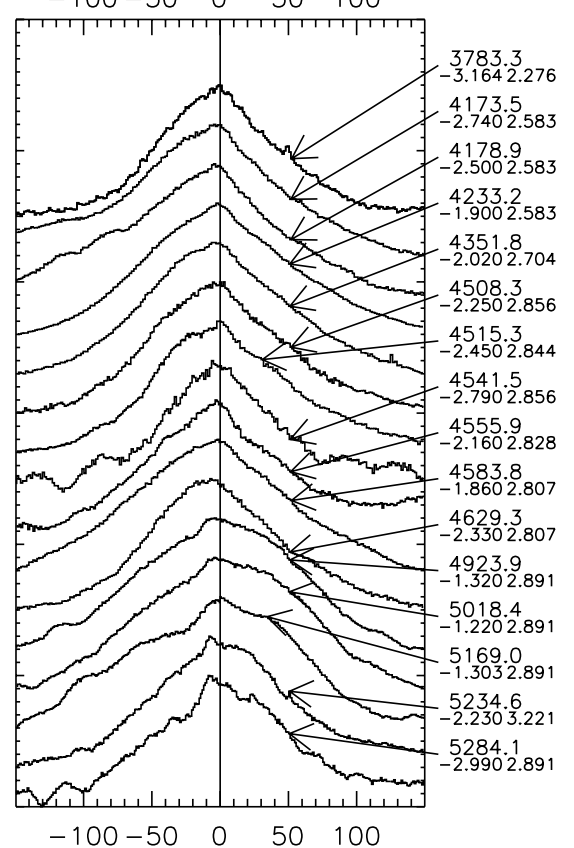

Fig. 7. Examples of $\mathrm{Fe}_{\mathrm{I}}$ (top) and $\mathrm{Fe}$ II (bottom) lines in the spectrum of RU Lup shown on velocity scale ( $\mathrm{km} \mathrm{s}^{-1}$ ). The vertical scale is arbitrary. The left panels show lines originating from the same upper level. The central panels show the lines sharing the same lower level. The profiles on these panels are offset by the level of the minimum point. Numbers on the right give the wavelength in $\AA$ and $\log g f$ of the line. The right panels compares practically all strong unblended Fe lines in our spectra. The profiles are shifted and scaled to the interval between 0 and 1 and offset for better visibility. In addition to the oscillator strength we also give the excitation energy of the lower level in eV. The light line in the lower left panel is shown as an illustration to compare shapes of lines sharing common upper levels. This line represents the profile of the $4178.9 \AA$ line scaled by the ratio of Einstein $A_{\text {ul }}$ probabilities (a factor of 1.84). In the optically thin case this profile should coincide with that of the $4555.9 \AA$ Aine (see also Sect. 4.3).

smallest structures of the accretion process. We present timeseries of some of these emission lines in Figs. 9, 10 and 11.

We did not include the $\mathrm{H} \alpha$ line in our analysis because the formation region of this line is much larger than the accretion region itself, and the line shape is sensitive to many more parameters than are relevant for the accretion process. On the other hand the shape and evolution of the all Balmer lines (at least up to H6, but most likely even further) is strik- ingly identical: during the first night, the lines show a doublepeaked profile with a separation of about $100 \mathrm{~km} \mathrm{~s}^{-1}$, while during the second night only a broad and flat profile is observed. The time-scale of evolution of the Balmer lines appears to be much longer than the typical time-scale of an accretion event, which suggests that these lines are formed in a large physical volume. We see some intensity variations of the Balmer lines on short time scales, notably $\mathrm{H} \beta$. 

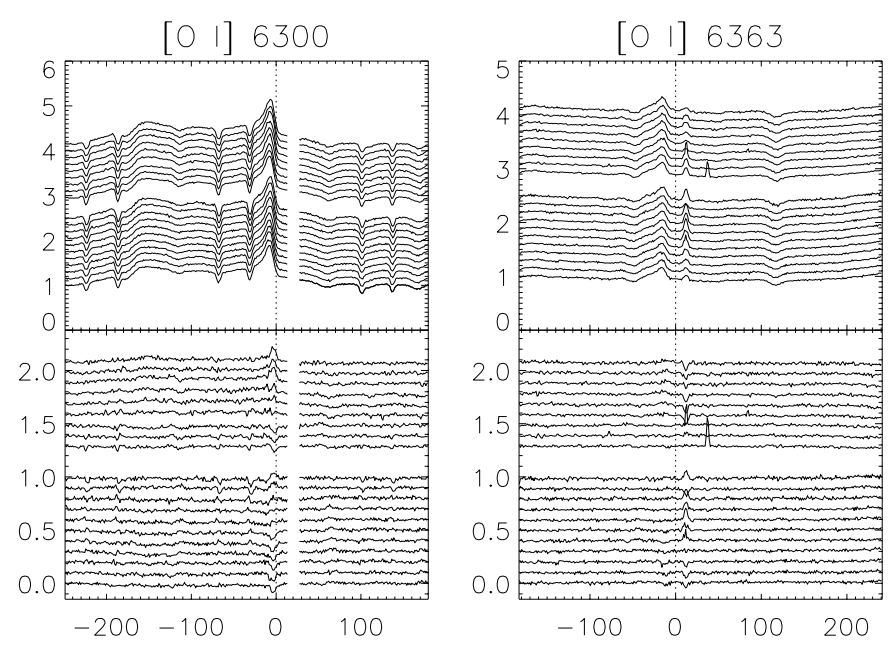

Fig. 8. Evolution of the observed line profiles of [O I] $6300 \AA$ and $6363 \AA$. The upper panel shows the observed profiles, the lower panel the difference of each profile with respect to the mean of all profiles. The sequence of the profiles reflects the order of observation, with the first spectrum at the bottom. The two nights of observations are separated by an extra offset. Units of the horizontal axis are $\mathrm{km} \mathrm{s}^{-1}$. The telluric component of [O I] $6300 \AA$ has been left out.
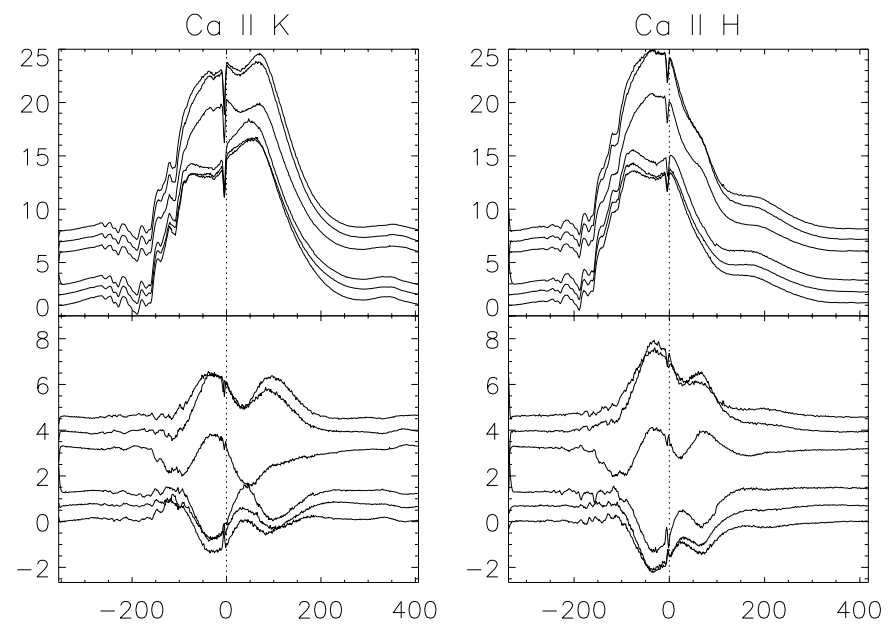

Fig. 9. As in Fig. 8, but for the $\mathrm{Ca}$ II $\mathrm{H}$ and $\mathrm{K}$ lines. An interstellar absorption line is clearly present.

The Ca II $\mathrm{H}$ and $\mathrm{K}$ lines behave very similar to the Balmer lines. Also here, we see a relatively large change in the overall line shape between the two nights, and the time-scale of changes in the line shape is considerably longer than the timescale of accretion events, suggesting the same conclusion about the formation region of $\mathrm{Ca}$ II $\mathrm{H}$ and $\mathrm{K}$ as for the Balmer lines. In addition, the $\mathrm{Ca}$ lines show blueshifted absorption features typical for outflow.

The shape of the He I (5876 ̊) line in RU Lup behaves in a different fashion than other strong emission lines. Because of its strength and width, this line is particularly suited for modelling by decomposition in different components. We decomposed the line in three Gaussian components, a core $\left(\sim 20 \mathrm{~km} \mathrm{~s}^{-1}\right)$, an intermediate- $\left(\sim 70 \mathrm{~km} \mathrm{~s}^{-1}\right)$ and a highvelocity component $\left(\sim 130 \mathrm{~km} \mathrm{~s}^{-1}\right)$, as shown in Fig. 12. For each observation we determined the three fundamental proper-
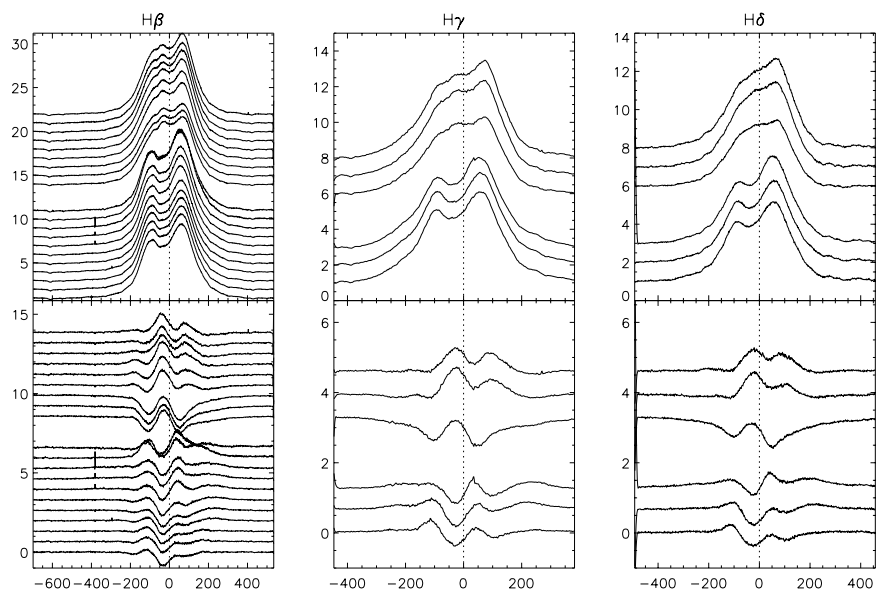

Fig. 10. As in Fig. 8, but for the $\mathrm{H} \beta, \mathrm{H} \gamma$ and $\mathrm{H} \delta$ lines. The Balmer lines behave very similar to each other, but also similar to the lines in Fig. 9.
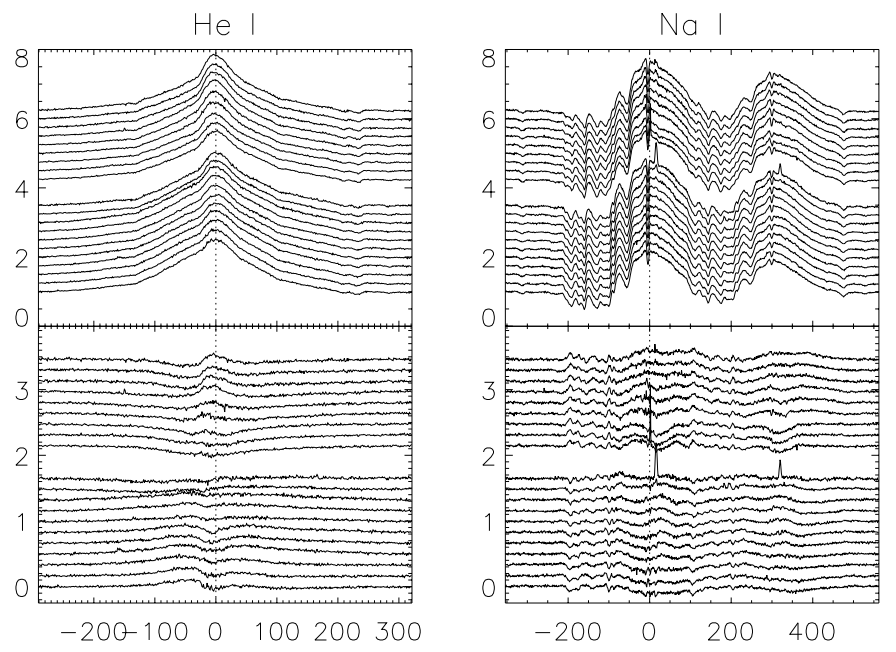

Fig. 11. As in Fig. 8, but for the $\mathrm{He}$ I and $\mathrm{Na}$ I $\mathrm{D}$ lines. Note the interstellar absorption lines and the appearance of telluric $\mathrm{Na}$ I emission at the end of the first night.

ties of each Gaussian component (see Fig. 13). There does not seem to be any correlation between these components and accretion events or the modulation due to rotation, except maybe for the strength of the broad component.

Of course, analysis of this line should be complemented with the line profile evolution from Fig. 11. From this figure it is evident that the blue and red wing of the broad component behave differently. Before and during the accretion events, there is an enhanced emission in the red wing. After the accretion events, there are signatures of absorption by outflowing gas at velocities typical of a heated corona, while there is an enhancement of line emission at near-zero velocities, as is reflected by the growing strength of the narrow component.

The Na I D doublet shows signs of absorption in the blue wing, typical for CTTSs with strong outflows. Variability of these absorption features is not correlated to the accretion activity. The line core, which is formed in the shock region, follows the accretion activity closely. 


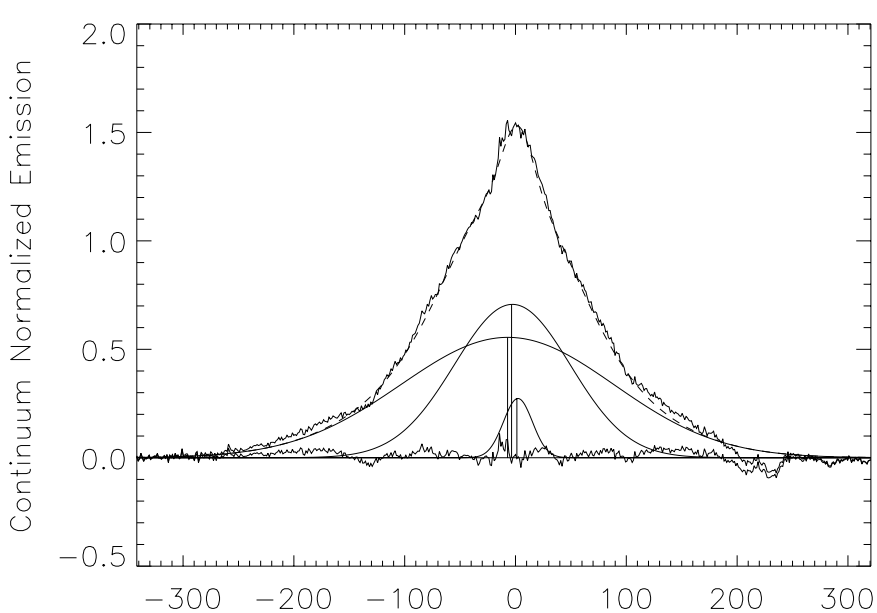

Fig. 12. Decomposition of the He I $5876 \AA$ line by least-squares fitting the observed profile (black) with three Gaussian components reproduces the line shape very well. Units of the horizontal axis are $\mathrm{km} \mathrm{s}^{-1}$. The residual is also shown.

\section{Discussion}

\subsection{Nature of the accretion process}

A broad range of different formation regions and mechanisms allows us to detect variability on a variety of time-scales. We define the shortest time-scale of incoherent changes such that variations of individual spectroscopic diagnostics on even shorter time-scales are smooth and predictable. This shortest time-scale for coherent changes is of the order of 1 hour (see for example Figs. 5, 9, 10 and 11). This is an important result, because it means the acquired dataset allows us to unambiguously investigate all transient phenomena in the accretion processes in CTTS, or at least RU Lup,

Most of our spectroscopic diagnostics vary on the derived time-scale. Because this time-scale is far less than the rotational period of RU Lup, this means that variations in these diagnostics reflect real changes in the stellar atmosphere and the circumstellar environment. This has the following implications:

- Variability on short time-scales has to be the result of changes in the mass accretion rate, rather than due to rotational modulation. This is strongly supported by variability in shape and strength of the strongest emission lines, which are formed in or close to the accretion region.

- Variability in the filling factor and the temperature of the accretion region is due to the fact that that the size and distribution of accretion regions is not constant in time.

In addition we often observe variability across the line profile, where the red and blue wing of an emission line do not correlate. Together with the previous two conclusions this means that it is not possible to describe the accretion process in RU Lup in terms of a star with its rotational axis perpendicular to the accretion disk and with an axisymmetric accretion flow.

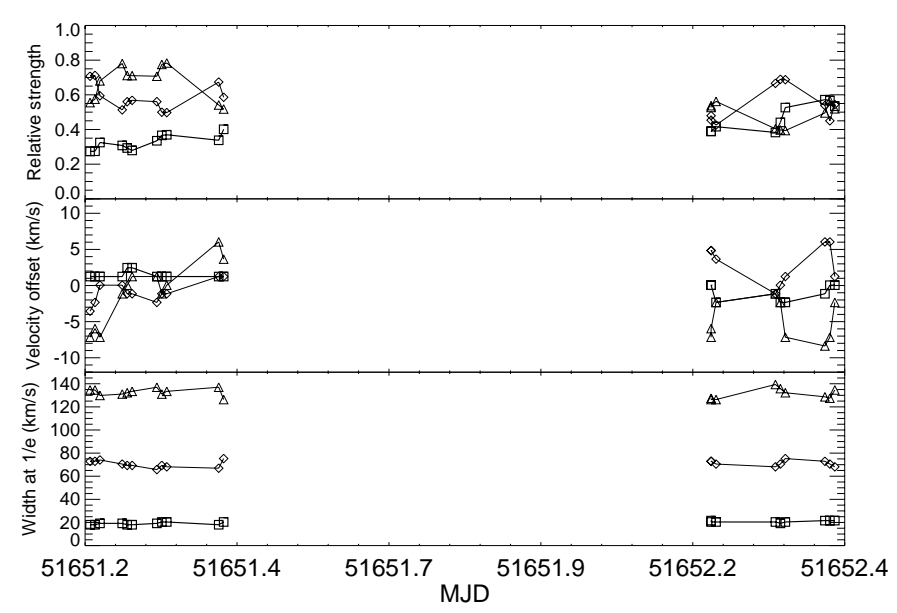

Fig. 13. Evolution of the fundamental parameters of the three Gaussian components of the $\mathrm{He}_{\mathrm{I}} 5876 \AA$ line during the two nights. The panels show the strength, position and width of each component. The squares, diamonds and triangles represent the narrow, intermediate and broad component respectively.

\subsection{Structure of the mass outflow}

The emission lines of $\mathrm{Na}$ I D and $\mathrm{Ca}$ II $\mathrm{H}$ and $\mathrm{K}$ are clearly accompanied by blue-shifted absorption features. The velocities corresponding to these features are as high as $250 \mathrm{~km} \mathrm{~s}^{-1}$ and indicate a strong mass outflow towards the observer. The shape and location in velocity space of these absorption features correspond well across these lines. More interestingly, the shape of these profiles change in a coherent fashion during the observing run. The profiles and a comparison of the first and last spectrum of Na I D2 are shown in Fig. 14. Also from the time-series of $\mathrm{Na} \mathrm{I}$ in Fig. 11 one can clearly follow the evolution of the outflow. The column density of outflowing matter is variable over a short time intervals. For example, the feature between -180 and $-210 \mathrm{~km} \mathrm{~s}^{-1}$ changes with as much as $60 \%$ in the course of the two nights of our observing run, reflecting that the outflow is of a strongly inhomogeneous nature. This feature alone corresponds to a column density of $N(\mathrm{Na} \mathrm{I}) \approx 5 \times 10^{11}$ atoms $\mathrm{cm}^{-2}$.

\subsection{Interstellar absorption and extinction}

Current estimates of the interstellar absorption and extinction available in the literature are of the order of $A_{V} \approx 1^{\mathrm{m}}$. These estimates rely on the assumption that RU Lup is a typical CTTS, embedded in a cloud of dust and gas, and that the extinction can be determined from reddening. However, for CTTS reddening alone may not be a good measure for extinction because of the dusty nature of the star and the presence of radiation from other sources such as accretion. Also, a picture of RU Lup as being observed pole-on is emerging. If accretion is occurring in a polar fashion, the assumptions on which existing estimates rely may not be valid. With our high-resolution and high-SNR spectra, we are for the first time able to measure the extinction directly from interstellar line absorption.

The equivalent width of the interstellar absorption line of Na I D2 (5890 ̊, visible on the right edge of Fig. 14) as measured from our data is $18.4 \mu \AA$. Under the assumption that the 


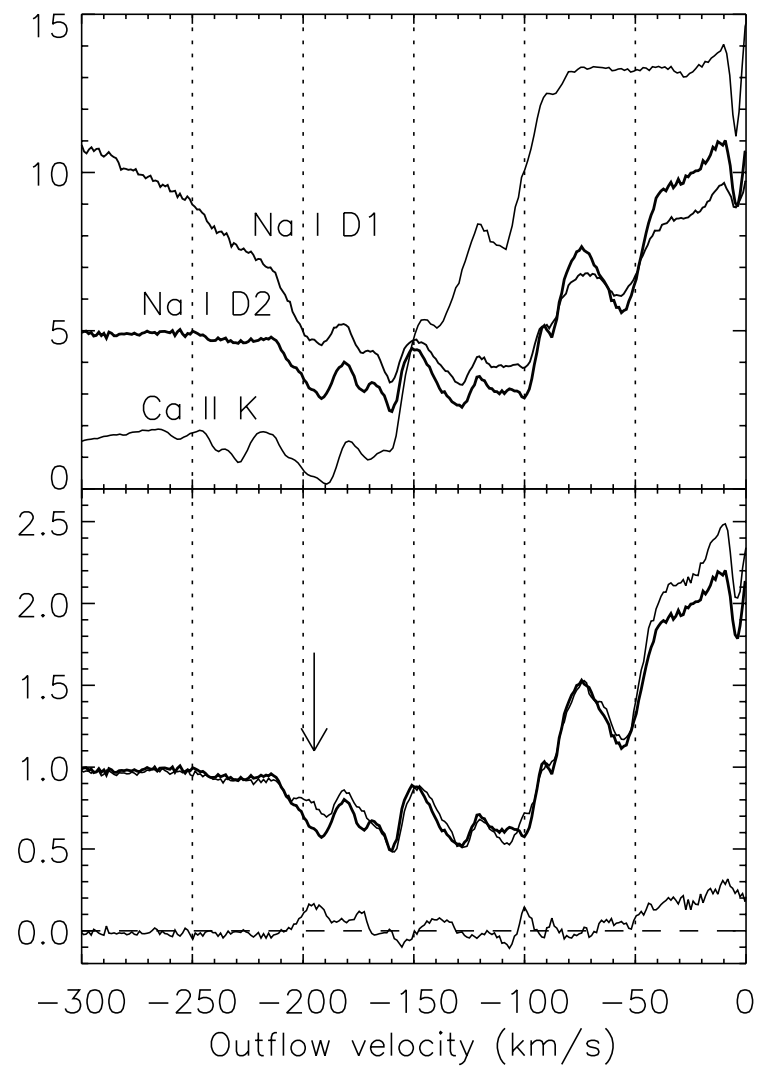

Fig. 14. Absorption features in the blue wing of the $\mathrm{Na}$ I $\mathrm{D}$ and $\mathrm{Ca}$ II $\mathrm{H}$ and $\mathrm{K}$ lines are very similar. The upper panel shows three of these lines. For the purpose of comparison, the two $\mathrm{Na}$ I $\mathrm{D}$ lines are scaled up by a factor of 5 relative to the $\mathrm{Ca}$ II $\mathrm{K}$ line. The bottom panel shows the first spectrum of the first night and the last spectrum of the second night of the NaID2 line, as well as their difference, illustrating the level of variability in the outflow. Note the excellent agreement of the profiles on the blue side of the line. The arrow indicates the feature which is mentioned in Sect. 5.2.

line is formed under optically thin conditions, which is applicable for this line, this corresponds to a column density of $N(\mathrm{Na}$ I $)=9.1 \times 10^{10}$ atoms $\mathrm{cm}^{-2}$. Following Welty \& Hobbs (2001) this corresponds to $N(\mathrm{H})=6.3 \times 10^{19} \mathrm{~cm}^{-2}$. This is a very low column depth for interstellar absorption; according to Burstein \& Heiles (1978) the corresponding colour excess is $-0.03<E(B-V)<0.06$. With an interstellar extinction of, in the worst case, $A_{V}=0.2$, we conclude that there is hardly any material other than ordinary interstellar matter along the line of sight; this also gives a very rough upper limit on the distance of $200 \mathrm{pc}$. Because this implies that there seems to be no obscuration of the accretion region, estimates of the accretion rate based on measurements of the accretion luminosity are most likely correct.

\subsection{Rotational period}

Determining the rotational period of a star known to exhibit strong erratic spectroscopic and photometric variability is not simple; the only photometric period reported for RU Lup is 3.7 days (Hoffmeister 1958, 1965) More recent investigations such as Giovannelli et al. (1991), using Fourier analysis on a dataset with a time base of several years, never confirmed this or any other period. The only estimate not based on long-term photometry comes from Lamzin et al. (1996). Assuming corotation of the star and the inner accretion disk, they determined a rotational period of RU Lup of 0.8 days.

Our observations do not allow a rotational period to be derived. However, it is possible to obtain constraints on the period and inclination from the projected rotational velocity:

$P<\frac{2 \pi R_{*}}{v \sin i}$.

Our measurement of the projected rotational velocity $v \sin i$ of $9.1 \pm 0.9 \mathrm{~km} \mathrm{~s}^{-1}$ and the estimated $R_{*} \approx 2.6 R_{\odot}$ gives an absolute upper limit on $P$ of 13.1 days. If the estimate of $P \approx 0.8$ days of Lamzin et al. is correct, this implies an inclination of $i \approx 3.5^{\circ}$ and a rotational velocity $v \approx 160 \mathrm{~km} \mathrm{~s}^{-1}$. Even the more moderate estimate of Hoffmeister of 3.7 days gives a result of $i \approx 16^{\circ}$ and $v \approx 35 \mathrm{~km} \mathrm{~s}^{-1}$.

\subsection{Is RU Lup observed pole-on?}

The conclusion of the previous paragraph suggests that RU Lup is observed at a low inclination angle. In our set of observations we find several indications that the line of sight is close to the direction of outflow:

- The overall shape of the forbidden emission line [O I] suggests an outflow towards the observer. Following the model of Edwards et al. (1987), [O I] lines are formed in a azimuthally symmetric latitude-dependent wind originating at the poles of the star. The accretion disk then acts as an opaque screen occulting the far side of the outflow. The overall shape of these lines then can be an indicator of the inclination of the system with respect to the observer's line of sight. The [O I] 6300 and $6363 \AA$ lines share the same formation region and the profiles reflect a time-integrated profile of the wind as the wind is optically thin. Our observations are in agreement with this model; the shape of both lines is identical, and no red-shifted emission can be seen. In our spectra of [ $\left.\mathrm{O}_{\mathrm{I}}\right]$ we find outflow velocities ranging from -200 to $0 \mathrm{~km} \mathrm{~s}^{-1}$. A study of outflow velocities of CTTS by Ardila et al. (2002) shows that RU Lup exhibits one of the most extreme blueshifts in their sample. To observe such a large range of outflow velocities, the line of sight should be along the outflow.

- Strong, correlated blue-shifted absorption features in the $\mathrm{Na}$ I D and $\mathrm{Ca}$ II $\mathrm{H}$ and $\mathrm{K}$ lines show a high-speed flow of matter towards the observer. Variability in these absorption features on short time scales suggests the line of sight to be close to the direction of outflow. There are no red-shifted absorption features.

In the frame of the magnetospheric accretion model, the outflow is collimated and directed along the rotational axis. Together with the arguments presented above, this is consistent with RU Lup being observed at low inclination. Also the very low interstellar extinction is inconsistent with a high inclination angle $\left(i>45^{\circ}\right)$. 


\subsection{Duplicity?}

It has been suggested that CTTS with extremely high accretion rates, such as RU Lup, might be accompanied by a faint secondary star. Recently periodic radial velocity variations were discovered by Petrov et al. (2001) for RW Aur A, another strongly accreting CTTS. The presence of a close companion could explain the mechanism of clearing the inner accretion region and feeding the accretion flow. An alternative explanation is that the magnetic dipole is inclined with respect to the accretion disk, inducing periodic changes in the visibility of the accretion region. Detailed inspection of our spectra of RU Lup shows no evidence of periodic changes of radial velocity. Of course, a low inclination of the system could make it impossible to detect a secondary component over two nights of observations.

\section{Conclusions}

During two nights in April 2000 we obtained spectra of high quality, in terms of SNR as well as in terms of spectral resolution, spectral coverage and short integration times, of $12 \mathrm{~T}$ Tauri stars. These spectra allowed us to perform a simultaneous analysis of a large number of different diagnostics during the two nights. We developed dedicated routines for reducing the observations and for determining specific properties of T Tauri stars such as the veiling factor.

During the first analysis of these data we concentrated on the star RU Lup. For this highly active and variable star we were able to separate the photospheric spectrum from the superimposed veiling continuum and infall- and outflow-related emission lines, which makes it possible to constrain the fundamental stellar properties such as $T_{\text {eff }}, \log g$, and $v \sin i$. The evolution of the veiling continuum and other spectral features of the accretion process shows that there is a shortest time scale of incoherent changes. From our observations we derive this time-scale to be of the order of 1 hour. Because this time-scale is far shorter than the rotational period, this has important implications on the geometry and nature of the accretion process. Variability on this time-scale throughout the spectrum leads us to believe that the accretion process cannot be described in terms of an axisymmetric model with a constant accretion rate. Either the accretion rate is variable or the rotational axis of the star is inclined with respect to its disk. Unfortunately, at this point, we cannot discriminate between these two models. On the other hand, the existence of such a time-scale implies that hourly observations during one or several rotational periods is optimal for studying the accretion process.

The emission lines of metals are a very promising tool for studying the accretion flow. They are numerous, mostly optically thin, and formed throughout the whole accretion region. The shape of the line profile is determined by the velocity distribution. Also, the intrinsic profile is quite narrow. The variability of e.g. iron lines is correlated with changes in veiling for both neutral and ion spectra. Previous attempts to synthesise these lines, even with simplified radiation transfer models, produced very encouraging results and the main task now is to determine the relation between the shape of the metal emission profiles and the geometry and velocity in the accretion flow.

The strongest emission lines of RU Lup exhibit night-tonight variability, but the changes are not correlated to the accretion process, which leads us to reject the idea that these lines are only generated in the accretion region. The blue wings of some of these lines clearly contain absorption features of outflowing matter. The shape and evolution of these features agrees very well across different species. The coherent variability leads us to the conclusion that the outflow is not homogeneous.

Outflow is also reflected in the presence of forbidden emission lines. Although these lines are formed under optically thin conditions, and therefore represent a time-integrated profile, variability is observed for the component with the lowest velocity.

The distance of RU Lup is difficult to determine. We provide a new estimate based on line absorption by interstellar matter.

A number of arguments indicate that RU Lup is most probably observed pole-on. We come to this conclusion by combining information of different diagnostics: the short rotational period, the shape of the forbidden emission lines, the shape and swift variations of absorption features related to the outflow and a relatively low amount of circumstellar and interstellar matter in the line of sight. Unfortunately this means that it is not possible to determine whether RU Lup has a close companion. We did not find (quasi-)periodic velocity variations in the star's photospheric absorption lines. Observing RU Lup poleon also makes it difficult to investigate the three-dimensional geometry.

\section{Future work}

Analysis of our spectra of T Tauri stars is far from finished. We expect to learn more about the three-dimensional accretion geometry by using our developed methods on similar stars that are not observed pole-on. The qualitative analysis of line variability needs to be completed with more quantitative analyses such as investigations of the equivalent widths of Fe emission lines with respect to their transition probabilities. Our main goal is to understand the nature of the accretion process and its threedimensional structure.

Finally we hope to be able to complete our dataset with observations on a longer time base. Such observations will allow us to generate a model that takes into account both variations on the shortest time scales (accretion) as well as modulation due to stellar rotation. At the moment we cannot separate these processes. When covering an entire rotational period we will be able to complete our analysis with surface maps using Doppler Imaging.

Acknowledgements. We would like to thank P. S. Barklem for correcting and commenting the article during the final stages of this paper. We are also grateful for the useful comments of G. Gahm and the anonymous referee that have improved this paper. 


\section{References}

Appenzeller, I., Krautter, J., \& Jankovics, I. 1983, A\&AS, 53, 291 Ardila, D. R., \& Basri, G. 2000, ApJ, 539, 834

Ardila, D. R., Basri, G., Walter, F. M., et al. 2002, ApJ, 567, 1013

Asplund, M., Gustafsson, B., Kiselman, D., et al. 1997, A\&A, 318, 521

Ballester, P., Modigliani, A., Boitquin, O., et al. 2000, The Messenger, 101,31

Basri, G., Marcy, G. W., \& Valenti, J. A. 1992, ApJ, 390, 622

Bertout, C., Basri, G., \& Bouvier, J. 1988, ApJ, 330, 350

Bertout, C. 1989, ARA\&A, 27, 351

Bertout, C., Robichon, N., \& Arenou, F. 1999, A\&A, 352, 574

Burstein, D., \& Heiles, C. 1978, ApJ, 225, 40

Calvet, N., \& Gullbring, E. 1998, ApJ, 509, 802

D'Odorico, S., Cristiani, S., Dekker, H., et al. 2000, Proc. SPIE, 4005, 121

Edwards, S., Cabrit, S., Strom, S. E., et al. 1987, ApJ, 321, 473

Gahm, G. F., Nordh, H. L., Olofsson, S. G., \& Carborg, N. C. J. 1974, A\&A, 33, 399

Gauvin, L. S., \& Strom, K. M. 1992, ApJ, 385, 217

Ghosh, P., \& Lamb, F. K. 1979, ApJ, 234, 296

Giovannelli, F., Errico, L., Vittone, A. A., \& Rossi, C. 1991, A\&AS, 87,89

Guenther, E. W., \& Emerson, J. P. 1997, A\&A, 321, 803

Gullbring, E. 1994, A\&A, 287, 131

Gullbring, E., Petrov, P. P., Ilyin, I., et al. 1996, A\&A, 314, 835

Gullbring, E., Hartmann, L., Briceño, C., \& Calvet, N. 1998, ApJ, 492, 323

Hamann, F., \& Persson, S. E. 1992, ApJS, 82, 285

Hartigan, P., Hartmann, L., Kenyon, S., \& Hewett, R. 1989, ApJS, 70, 899

Hartigan, P., Edwards, S., \& Ghandour, L. 1995, ApJ, 452, 736

Hartmann, L., Boss, A., Calvet, N., \& Whitney, B. 1994, ApJ, 430, L49

Hartmann, L., Calvet, N., Gullbring, E., \& D’Alessio, P. 1998, ApJ, 495,385

Herbig, G. H., \& Bell, K. R. 1988, Lick Observatory Bull., No. 1111 Hoffmeister, C. 1958, Veröff. Sternwarte Sonneberg, 3, 333

Hoffmeister, C. 1965, Veröff. Sternwarte Sonneberg, 6, 91
Imhoff, C. L., \& Giampapa, M. S. 1980, ApJ, 239, L115

Johns-Krull, C. M., \& Hatzes, A. P. 1997, ApJ, 487, 896

Johns-Krull, C. M., \& Basri, G. 1997, ApJ, 474, 433

Johns-Krull, C. M., \& Valenti, J. A. 2000, in Stellar Clusters and Associations: Convection, Rotation, and Dynamos, ed. R. Pallavicini, G. Micela, \& S. Sciortino, ASP Conf. Ser., 198, 371

Johnstone, R. M., \& Penston, M. V. 1987, MNRAS, 227, 797

Joy, A. H. 1945, ApJ, 102, 168

Kurucz, R. L., Furenlid, I., Brault, J., \& Testerman, L. 1984, National Solar Observatory Atlas, No. 1 (Tucson: NSO)

Königl, A. 1991, ApJ, 370, L39

Kuschnig, R., Ryabchikova, T. A., Piskunov, N. E., Weiss, W. W., \& Gelbmann, M. J. 1999, A\&A, 348, 924

Kwan, J., \& Tademaru, E. 1995, ApJ, 454, 382

Lamzin, S. A., Bisnovatyi-Kogan, G. S., Errico, L., et al. 1996, A\&A, 306, 877

Lamzin, S. A., Stempels, H. C., \& Piskunov, N. E. 2001, A\&A, 369, 96

Lynden-Bell, D., \& Pringle, J. E. 1974, MNRAS, 168, 603

Marsh, T. R., \& Horne, K. 1988, MNRAS, 235, 269

Muzerolle, J., Calvet, N., \& Hartmann, L. 1998a, AJ, 116, 455

Muzerolle, J., Hartmann, L., \& Calvet, N. 1998b, AJ, 116, 455

Muzerolle, J., Calvet, N, \& Hartmann, L. 2001, ApJ, 550, 944

Piskunov, N. E., Kupka, F., Ryabchikova, T. A., Weiss, W. W., \& Jeffery, C. S. 1995, A\&AS, 112, 525

Piskunov, N., \& Valenti, J. 2002, A\&A, 385, 1095

Petrov, P. P., Gahm, G. F., Gameiro, J. F., et al. 2001, A\&A, 369, 993

Reipurth, B., Pedrosa, A., \& Lago, M. T. V. T. 1996, A\&AS, 120, 229

Rice, J. B., \& Strassmeier, K. G. 1996, A\&A, 316, 164

Smith, K. W., Lewis, G. F., Bonnell, I. A., Bunclark, P. S., \& Emerson, J. P. 1999, MNRAS, 304, 367

Strassmeier, K. G., Rice, J. B., Wehlau, et al. 1991, A\&A, 247, 130

Uchida, Y., \& Shibata, K. 1985, PASJ, 37, 515

Valenti, J., \& Piskunov, N. 1996, A\&AS, 118, 595

Valenti, J. A., Johns-Krull, C. M., \& Piskunov, N. E. 2001, in 11th Cambridge Workshop on Cool Stars, Stellar Systems and the Sun, ed. R. J. Garcia Lopez, R. Rebolo, \& M. Rosa Zapaterio Osorio, ASP Conf. Ser., 223, 1579

Welty, D. E., \& Hobbs, L. M. 2001, ApJS, 133, 345 